\title{
EXPONENTIAL LIMITING PRODUCTS IN BANACH ALGEBRAS
}

\author{
BY \\ B. O. KOOPMAN
}

1. Introduction. The formula of elementary calculus

$$
\lim _{n \rightarrow \infty}\left(1+\frac{a}{n}\right)^{n}=e^{a}
$$

generalizes easily to the theorem that if $\{a\}=\left\{a_{1}, a_{2}, \cdots\right\}$ is a sequence in which the limit

$$
m=\lim _{n \rightarrow \infty}\left(a_{1}+\cdots+a_{n}\right) / n
$$

exists and

$$
\left(\left|a_{1}\right|+\cdots+\left|a_{n}\right|\right) / n
$$

is bounded, then

$$
\lim _{n \rightarrow \infty} \prod_{s=1}^{n}\left(1+\frac{a_{s}}{n}\right)=e^{m}
$$

From this it follows almost trivially that if $\{b\}=\left\{b_{1}, b_{2}, \cdots\right\}$ is a second sequence for which

$$
\lim _{n \rightarrow \infty} \prod_{s=1}^{n} b_{s}=p \neq 0
$$

exists, then

$$
\lim _{n \rightarrow \infty} \prod_{s=1}^{n}\left(b_{s}+\frac{a_{s}}{n}\right)=p e^{m} .
$$

Finally, these formulas subsist even when each $a_{s}$ in (1.4) and (1.6) is replaced by $a_{n}+c_{n, s}$, where

$$
\lim _{n \rightarrow \infty}\left(\left|c_{n, 1}\right|+\cdots+\left|c_{n, n}\right|\right) / n=0 .
$$

These results generalize immediately to cases where the denominator $n$ in (1.2)-(1.7) is replaced by $\omega_{n}$, the $n$th term of a sequence $\{\omega\}=\left\{\omega_{1}\right.$, $\left.\omega_{2}, \cdots\right\}$ of positive numbers increasing monotonically to infinity and with $\omega_{n+1} / \omega_{n}$ approaching unity. A theorem of this sort is used in a proof of the

Presented to the Society, December 28, 1949; received by the editors November 4, 1949. 
Central Limit Theorem of probability.

All these theorems are proved immediately by taking logarithms of the product in (1.4), and applying a suitable form of Taylor's Remainder Theorem of the second order, and then making an obvious evaluation of the sum of remainders. One uses the fact that since

$$
\frac{a_{n}}{\omega_{n}}=\left(\frac{1}{\omega_{n}} \sum_{i=1}^{n} a_{i}-\frac{1}{\omega_{n-1}} \sum_{i=1}^{n-1} a_{i}\right)+\left(1-\frac{\omega_{n-1}}{\omega_{n}}\right) \frac{1}{\omega_{n-1}} \sum_{i=1}^{n-1} a_{i},
$$

$a_{n} / \omega_{n} \rightarrow 0$ as $n \rightarrow \infty\left(^{1}\right)$.

The object of the present paper is to extend all these theorems to the case where the elements of $\{a\}$ and $\{b\}$ belong to a Banach algebra $\Re$ containing the identity. Since multiplication in $\Re$ is in general neither commutative nor invertible, the process of taking logarithms is no longer available; and the proofs are no longer strictly elementary. Formula (1.4) (with $n$ replaced by $\omega_{n}$ ) remains valid, $e^{m}$ being defined by the infinite series. But (1.6) has to be modified by replacing $m$ by the quantity

$$
M=\lim _{n \rightarrow \infty}\left(a_{1} B_{2}+a_{2} B_{3}+\cdots+a_{n} B_{n+1}\right) / n
$$

where

$$
B_{i}=\lim _{\nu \rightarrow \infty} b_{i} b_{i+1} \cdots b_{i+\nu-1}
$$

(under suitable hypothesis as to these limits).

One example of our results arises in the case where $\Re$ is the ring of $r$-rowed square matrices (the singularity or nonsingularity of which is irrelevant). Another example occurs when $\Re$ is the class of bounded linear transformations in Hilbert space. And still other examples can be given. The case of matrices not only makes contact with the theory of iteration of linear transformations in $r$-space, but is needed for the proof of the Law of Small Numbers in nonstationary Markoff processes, established recently by the author $\left({ }^{2}\right)$. Of course the extension of (1.1) to Banach algebras is trivial: it is contained in well known results in group theory.

2. The Banach algebra. We recall that a Banach algebra is a system $\Re$ possessing the following four properties $\left({ }^{3}\right)$ :

(i) $\Re$ is a ring (in general, noncommutative).

(1) A more general theorem of this sort is contained in the author's note: Necessary and sufficient conditions for Poisson's distribution, Proceedings of the American Mathematical Society vol. 1 (1950) pp. 813-823.

(2) B. O. Koopman, The law of small numbers in Markoff chains, Trans. Amer. Math. Soc. vol. 70 (1951) pp. 277-290. Actually the author was led to the results of the present paper by his attempt to solve this problem of probability.

(3) Cf. E. Hille, Functional analysis and semi-groups, Amer. Math. Soc. Colloquium Publications, vol. 31, New York, p. 12. 
(ii) $\Re$ has the set of real (or complex) numbers as multiplicative operators.

(iii) $\Re$ is normed. The numerical function $N(a)$ is defined for all $a$ of $\Re$ and has the usual properties of a norm, among others, $N(a b) \leqq N(a) N(b)$.

(iv) $\Re$ is complete with respect to convergence in the norm $N$. Finally, for our purposes, we shall add the requirement that

(v) $\Re$ contains the identity $1(1 a=a 1=a$ for all $a \in \Re)$.

A first example of such an $\Re$ is the class of all $r$-rowed square matrices of real (or of complex) elements, with the usual definition (Frobenius-Wedderburn)

$$
N(a)=\left(\operatorname{trace} a a^{*}\right)^{1 / 2}=\left(\sum_{i=1}^{r} \sum_{j=1}^{r}\left|\alpha_{i j}\right|^{2}\right)^{1 / 2},
$$

where $\alpha_{i j}$ is the general element of $a$. Here, convergence $a(n) \rightarrow a$ amounts to convergence of corresponding elements, $\alpha_{i j}(n) \rightarrow \alpha_{i j}$.

A second example is when $\Re$ is the class of bounded (namely, continuous) linear operators of Hilbert space: those operators $a$ for which (parenthesis denoting the fundamental bilinear form):

$$
(a \phi, a \phi) /(\phi, \phi) \leqq G<\infty
$$

for all elements $\phi \neq 0$ of the Hilbert space. $G$ is independent of $\phi$ but depends on $a$. The non-negative square root of its greatest lower bound is a well known definition of $N(a)$.

A third class of examples arises as follows: Let $D$ be a domain in the space of one or more variables belonging to the (real or complex) scalar multiplier field of a given $\Re$. Consider the functions defined on the common domain $D$ and having values in the range $\Re$. Those of bounded norm over $\Re$ may be taken as the elements of a new $\Re$, sums and products being defined by adding and multiplying the functions, and the norm defined as the least upper bound over $D$ of the norm of their values on $D$. More generally, measurability and summability of such functions being definable over $D$, the new $\Re$ may be defined as the class of, say, summable-square functions, the new norm being the square root of the integral over $D$ of the square of the absolute value of the corresponding function $\left({ }^{4}\right)$. Extensions are obvious. Obvious also is the extension of the first example to the case where the matrix elements $\alpha_{i j}$ are functions of the proper type defined over $D$.

Further examples, involving continuous transition probabilities, can be given.

We define the exponential of any element $c$ of $R$ by the series

$$
e^{c}=\sum_{n=0}^{\infty} \frac{c^{n}}{n !} .
$$

That the series always converges is seen from the relation

(4) Cf. E. Hille, loc. cit., chap. III. 


$$
N\left(\sum_{k=n+1}^{n+p} \frac{c^{k}}{k !}\right) \leqq \sum_{k=n+1}^{n+p} \frac{N(c)^{k}}{k !},
$$

together with the convergence of the ordinary exponential series for $e^{N(c)}$. If $a b=b a$, simple power series manipulations show that $e^{a+b}=e^{a} e^{b}$; but when $a b \neq b a$, this is no longer valid $\left(^{5}\right)$.

We close this section by remarking that all the theorems of this paper concerning limits of products of elements of $\Re$ are equally valid if the order of all factors are simultaneously reversed. This is the result of the skew automorphism $x \rightarrow x^{\prime}$ of $\Re$ into itself, which causes

$$
\left.(a+b)^{\prime}=a^{\prime}+b^{\prime}, \quad(a b)=b^{\prime} a^{\prime}, \quad(\lambda a)^{\prime}=\lambda a^{\prime} \quad \text { ( } \quad \text {, scalar }\right) .
$$

3. The averaging sequence. By an averaging sequence we shall mean any sequence $\{\omega\}$ of real numbers having the three following properties:

$$
\begin{gathered}
0<\omega_{1} \leqq \omega_{2} \leqq \omega_{3} \leqq \cdots ; \\
\omega_{n} \rightarrow \infty \text { as } n \rightarrow \infty ; \\
\lim _{n \rightarrow \infty} \omega_{n+1} / \omega_{n}=1 .
\end{gathered}
$$

The simplest example of an averaging sequence is the sequence of positive integers, or any arithmetic progression of positive terms. Another example is the sequence $\omega_{n}=n^{c}$, where $c$ is any positive constant. And we may have $\omega_{n}$ $=\log n$, or $\omega_{n}=n^{c} \log n$ (for $n>1$ ), and so forth. An example which occurs in a proof of the Central Limit Theorem of probability is the following: Let $\left\{b_{1}, b_{2}, \cdots\right\}$ be any sequence of positive constants (in the application cited, they are the variances of the variates added), having the property that for each $s$,

$$
b_{s} /\left(b_{1}+\cdots+b_{n}\right) \rightarrow 0
$$

uniformly over the set $(0<s<\infty)$ as $n \rightarrow \infty$. Then the sequence $\{\omega\}$

$$
\omega_{n}=b_{1}+\cdots+b_{n}
$$

evidently satisfies conditions (3.1), (3.2), (3.3).

But the sequence $\omega_{n}=c^{n}(c \neq 1)$ is evidently not an averaging sequence. Nor is $\omega_{n}=b_{1}+\cdots+b_{n}$ an averaging sequence if the $b$-series satisfies a Hadamard gap condition.

We shall write, for any positive integers $n, k$,

$$
\Delta \omega_{n}=\omega_{n+1}-\omega_{n}, \quad \Delta \omega_{n}^{k}=\stackrel{k}{\omega_{n+1}^{k}}-\stackrel{k}{\omega_{n}^{k}} .
$$

Lемма I. For every averaging sequence $\{\omega\}$, and positive integer $k$,

(5) Cf. E. Hille, loc. cit., $\$ 5.18$. 


$$
\lim _{n \rightarrow \infty} \frac{1}{\omega_{n}^{k}} \sum_{i=1}^{n-1} \omega_{i}^{k-1} \Delta \omega_{i}=\frac{1}{k} .
$$

We have on the one hand

$$
\sum_{i=1}^{n-1} \Delta \omega_{i}^{k}=\sum_{i=1}^{n-1}\left(\omega_{i+1}^{k}-\omega_{i}^{k}\right)=\omega_{n}^{k}-\omega_{1}^{k}
$$

and, on the other hand,

$$
\begin{aligned}
\sum_{i=1}^{n-1} \Delta \omega_{i}^{k} & =\sum_{i=1}^{n-1}\left[\left(\omega_{i}+\Delta \omega_{i}\right)^{k}-\omega_{i}^{k}\right] \\
& =k \sum_{i=1}^{n-1} \omega_{i}^{k-1} \Delta \omega_{i}+\sum_{i=1}^{n-1} \sum_{j=2}^{k} C_{j}^{k \omega_{i}^{k-j}}\left(\Delta \omega_{i}\right)^{j}
\end{aligned}
$$

where $C_{j}^{k}=k ! / j !(k-j)$ ! is the binomial coefficient. Equating these expressions, and dividing by $k \omega_{n}^{k}$, we obtain

$$
\frac{1}{\omega_{n}^{k}} \sum_{i=1}^{n-1} \omega_{i}^{k-1} \Delta \omega_{i}=\frac{1}{k}-\frac{1}{k}\left(\frac{\omega_{1}}{\omega_{n}}\right)^{k}-\frac{1}{k} \sum_{j=2}^{k} C_{j}^{k} \sum_{i=1}^{n-1}\left(\frac{\omega_{i}}{\omega_{n}}\right)^{k-j}\left(\frac{\Delta \omega_{i}}{\omega_{n}}\right)^{j}
$$

In virtue of (3.2), equation (3.4) will be established if it can be shown that the last sum on the right approaches zero as $n \rightarrow \infty$.

For this purpose, we note that on account of (3.1),

$$
0 \leqq \sum_{i=1}^{n-1}\left(\frac{\omega_{i}}{\omega_{n}}\right)^{k-j}\left(\frac{\Delta \omega_{i}}{\omega_{n}}\right)^{j} \leqq \sum_{i=1}^{n-1}\left(\frac{\Delta \omega_{i}}{\omega_{n}}\right)^{j}
$$

Letting $i(n)$ denote the subscript of the (first) maximum $\Delta \omega_{i}$ in the set $\left(\Delta \omega_{1}, \cdots, \Delta \omega_{n}\right)$, and recalling that $j \geqq 2$, we see that

$$
\sum_{i=1}^{n-1}\left(\frac{\Delta \omega_{i}}{\omega_{n}}\right)^{j} \leqq\left(\frac{\Delta \omega_{i(n)}}{\omega_{n}}\right)^{j-1} \cdot \frac{1}{\omega_{n}} \sum_{i=1}^{n-1} \Delta \omega_{i} .
$$

Now

$$
\frac{1}{\omega_{n}} \sum_{i=1}^{n-1} \Delta \omega_{i}=\frac{1}{\omega_{n}}\left(\omega_{n}-\omega_{1}\right)=1-\frac{\omega_{1}}{\omega_{n}}
$$

and this approaches unity, in view of (3.2). It remains to show that $\Delta \omega_{i(n)} / \omega_{n}$ $\rightarrow 0$ as $n \rightarrow \infty$. There are two possibilities: $i(n)$, which increases with $n$ if it changes at all, may either remain finite or become infinite. In the former case the desired result is a consequence of (3.2). In the latter, we have, using (3.1),

$$
0 \leqq \frac{\Delta \omega_{i(n)}}{\omega_{n}}=\frac{\Delta \omega_{i(n)}}{\omega_{i(n)}} \cdot \frac{\omega_{i(n)}}{\omega_{n}} \leqq \frac{\Delta \omega_{i(n)}}{\omega_{i(n)}}=\frac{\omega_{i(n)+1}}{\omega_{i(n)}}-1 ;
$$


and the required approach to zero follows from (3.3). This completes the proof of the lemma.

As an immediate corollary, an application of (3.2) shows that

$$
\frac{1}{\omega_{n}^{k}} \sum_{\nu=k}^{n} \omega_{\nu-1}^{k-1} \Delta \omega_{\nu-1}=\frac{1}{k}+\zeta(n, k), \quad \lim _{n \rightarrow \infty} \zeta(n, k)=0 \quad(k \geqq 2) .
$$

4. Limits of the expansion terms. In this section, $\{\omega\}$ will be a fixed averaging sequence, and $\{a\}=\left\{a_{1}, a_{2}, \cdots\right\}$ an infinite sequence of elements of $\Re$, having the two following properties:

$$
\begin{array}{rlr}
\lim _{n \rightarrow \infty}\left[\frac{1}{\omega_{n}} \sum_{i=1}^{n} a_{i}\right] & =m, & \\
\frac{1}{\omega_{n}} \sum_{i=1}^{n} N\left(a_{i}\right) & \leqq \alpha<\infty & \text { for all } n .
\end{array}
$$

Here and throughout this paper we shall use the following notation for certain sums: If $x\left(i_{1}, \cdots, i_{k}\right)(1 \leqq k \leqq n)$ is any symbol (for example, a product) involving the subscripts $i_{1}, \cdots, i_{k}$, whether it represent an element of $\Re$ or an ordinary number, we shall denote by

$$
\sum_{(i)}^{n} x\left(i_{1}, \cdots, i_{k}\right)
$$

the sum over all sets $\left(i_{1}, \cdots, i_{k}\right)$ satisfying the inequalities

$$
1 \leqq i_{1}<i_{2}<\cdots<i_{k} \leqq n .
$$

There are obviously $C_{k}^{n}=n ! / k !(n-k) ! x$-terms in this sum. And we shall denote by

$$
\sum_{[i]}^{n} x\left(i_{1}, \cdots, i_{k}\right)
$$

the sum over all sets $\left(i_{1}, \cdots, i_{k}\right)$ satisfying the inequalities

$$
\begin{aligned}
1 & \leqq i_{\rho} \leqq n ; \quad i_{\rho} \neq i_{\sigma} \text { when } \rho \neq \sigma ; \\
\rho, \sigma & =1,2, \cdots, n .
\end{aligned}
$$

Evidently this sum has $P_{k}^{n}=n ! /(n-k) ! x$-terms. And if every $x\left(i_{1}, \cdots, i_{k}\right)$ is symmetric in the subscripts, then

$$
\sum_{(i)}^{n} x\left(i_{1}, \cdots, i_{k}\right)=\frac{1}{k !} \sum_{[i]}^{n} x\left(i_{1}, \cdots, i_{k}\right) .
$$

In the special case of the product of $k$ numerical factors not less than zero, 


$$
x\left(i_{1}, \cdots, i_{k}\right)=x_{i_{1}} \cdots x_{i_{k}}, \quad x_{i} \geqq 0 \quad(i=1, \cdots, n),
$$

we have at once

$$
\begin{aligned}
\sum_{(i)}^{n} x_{i_{1}} \cdots x_{i_{k}} & =\frac{1}{k !} \sum_{[i]}^{n} x_{i_{1}} \cdots x_{i_{k}} \\
& \leqq \frac{1}{k !} \sum_{i_{1}=1}^{n} \cdots \sum_{i_{k}=1}^{n} x_{i_{1}} \cdots x_{i_{k}} \\
& =\frac{1}{k !}\left[\sum_{i=1}^{n} x_{i}\right]^{k} .
\end{aligned}
$$

In dealing with noncommuting products, we shall use the symbol $\prod_{s=\rho}^{\sigma}$ to denote the product of factors running from left to right as $s$ goes from the bottom value $\rho$ to the top value $\sigma: \prod_{s=\sigma}^{\rho}$ would be the product of the same factors in reversed order.

By an obvious expansion, with the order of factors maintained, we obtain

$$
\prod_{s=1}^{n}\left(1+\frac{a_{s}}{\omega_{n}}\right)=\left(1+\frac{a_{1}}{\omega_{n}}\right) \cdots\left(1+\frac{a_{n}}{\omega_{n}}\right)=\sum_{k=0}^{n} p(n, k),
$$

where $p(n, 0)=1$, and, for $k \geqq 1$,

$$
p(n, k)=\frac{1}{\omega_{n}^{k}} \sum_{(i)}^{n} a_{i_{1}} \cdots a_{i_{k}} .
$$

In this section we shall take a first step towards establishing (1.2) in the present case by proving that $p(n, k) \rightarrow m^{k} / k$ ! as $n \rightarrow \infty$.

LEMмA II. For each integer $k>1$,

$$
\lim _{n \rightarrow \infty} \frac{1}{\omega_{n}^{k}} \sum_{\nu=k}^{n} \begin{gathered}
k-1 \\
\omega_{\nu-1}^{k} a_{\nu}
\end{gathered}=\frac{m}{k} .
$$

Writing, for each positive integral $\nu$,

$$
s_{\nu}=a_{1}+\cdots+a_{\nu}=\left(m+d_{\nu}\right) \omega_{\nu},
$$

we evidently have (using the subscript difference operation $\Delta$ ):

$$
\begin{gathered}
a_{\nu}=s_{\nu}-s_{\nu-1}=m \Delta \omega_{\nu-1}+\Delta\left(d_{\nu-1} \omega_{\nu-1}\right) \\
\lim _{\nu \rightarrow \infty} d_{\nu}=0
\end{gathered}
$$

the latter, on account of (4.1). Then we obtain

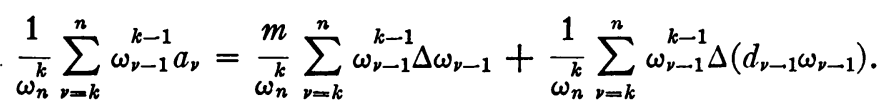


Now the first expression on the right approaches $m / k$ as $n \rightarrow \infty$, in virtue of Lemma I, (3.5). That the last expression approaches zero appears as follows:

By a rearrangement of terms (cf. the "summation by parts" of the calculus of finite differences), we get

$$
\begin{aligned}
\frac{1}{\omega_{n}^{k}} \sum_{\nu=k}^{n} \omega_{\nu-1}^{k-1} \Delta\left(d_{\nu-1} \omega_{\nu-1}\right) & =\left(\frac{\omega_{n-1}}{\omega_{n}}\right)^{k-1} d_{n}-\frac{\omega_{k-1}^{k} d_{k-1}}{\omega_{n}^{k}}+S(n, k), \\
S(n, k) & =\frac{1}{\omega_{n}^{k}} \sum_{\nu=k}^{n-1} d_{\nu} \omega_{\nu} \Delta \omega_{\nu-1}^{k-1} .
\end{aligned}
$$

Since the first two terms on the right in (4.10) approach zero as $n \rightarrow \infty$, on account of (3.2), (3.3), (4.9), we have but to show that $S(n, k) \rightarrow 0$.

Let $\epsilon>0$ be given, and $\mu=\mu(\epsilon)$ be an integer such that $\nu \geqq \mu$ implies that $N\left(d_{v}\right)<\epsilon$ (cf. (4.9)). As soon as $n>\mu+1$, we have (assuming, as we may, that $\mu>k)$

$$
N[S(n, k)] \leqq \frac{1}{\omega_{n}^{k}} \sum_{\nu=k}^{\mu-1} N\left(d_{\nu}\right) \omega_{\nu} \Delta \omega_{\nu-1}^{k-1}+\frac{\epsilon}{\omega_{n}^{k}} \sum_{\nu=\mu}^{n-1} \omega_{\nu} \Delta \omega_{\nu-1}^{k-1}
$$

Now the first expression on the right, being the sum of $\mu-k$ terms all independent of $n$, divided by the indefinitely increasing $\omega_{n}^{k}$, approaches zero as $n \rightarrow \infty$. For the second expression we obtain, after a rearrangement of terms,

$$
0 \leqq \frac{\epsilon}{\omega_{n}^{k}} \sum_{\nu=\mu}^{n-1} \omega_{\nu} \Delta \omega_{\nu-1}^{k \rightarrow 1}=\epsilon\left[\left(\frac{\omega_{n-1}}{\omega_{n}}\right)^{k}-\frac{\omega_{\mu} \omega_{\mu-1}^{k-1}}{\omega_{n}^{k}}-\sum_{\nu=\mu}^{n-2} \omega_{\nu}^{k-1} \Delta \omega_{\nu}\right] \leqq \epsilon\left(\frac{\omega_{n-1}}{\omega_{n}}\right)^{k} \rightarrow \epsilon
$$

This, in connection with (4.11), shows that

$$
0 \leqq \limsup _{n \rightarrow \infty} N[S(n, k)] \leqq \epsilon ;
$$

and since $\epsilon$ is arbitrary, it follows that $S(n, k) \rightarrow 0$; and the proof of Lemma II is complete.

LEMmA III. For each $k=0,1,2, \cdots$,

$$
\lim _{n \rightarrow \infty} p(n, k)=\frac{m^{k}}{k !} .
$$

This equation, trivial for $k=0$, coincides with (4.1) when $k=1$. It is proved by induction: Writing, for all $n, k$,

$$
p(n, k)=\frac{m^{k}}{k !}+z(n, k)
$$

we assume that $z(n, k-1) \rightarrow 0$ as $n \rightarrow \infty$, and shall prove that $z(n, k) \rightarrow 0(k \geqq 2)$. 
The evident relation

$$
\sum_{(i)}^{n} a_{i_{1}} \cdots a_{i_{k}}=\sum_{\nu=k}^{n}\left(\sum_{(i)}^{\nu-1} a_{i_{1}} \cdots a_{i_{k-1}}\right) a_{\nu}
$$

leads, with the use of (4.7), to the formula

$$
p(n, k)=\frac{1}{\omega_{n}^{k}} \sum_{\nu=k}^{n} p(\nu-1, k-1) \omega_{\nu-1}^{k-1} a_{\nu} .
$$

On replacing the $p$ 's by their expressions in (4.13), and solving the resulting equation for $z(n, k)$, one obtains

$$
\begin{aligned}
& z(n, k)=\frac{m^{k-1}}{(k-1) !}\left(\frac{1}{\omega_{n}^{k}} \sum_{\nu=k}^{n} \omega_{\nu-1}^{k-1} a_{\nu}-\frac{m}{k}\right)+R(n, k), \\
& R(n, k)=\frac{1}{\omega_{n}^{k}} \sum_{\nu=k}^{n} z(\nu-1, k-1) \omega_{\nu-1}^{k-1} a_{\nu} .
\end{aligned}
$$

Lemma II shows that the first expression on the right approaches zero as $n \rightarrow \infty$. It remains to show that $R(n, k) \rightarrow 0$.

Let $\epsilon>0$ be given. By the inductive assumption, there exists a $\mu$ (which we may and will take greater than $k+1$ ) such that $\nu \geqq \mu-1$ implies that $N[z(\nu-1, k-1)]<\epsilon$. Then we have

$$
N[R(n, k)] \leqq \frac{1}{\omega_{n}^{k}} \sum_{\nu=k}^{\mu-1} N[z(\nu-1, k-1)] \omega_{\nu-1}^{k-1} N\left(a_{\nu}\right)+\frac{\epsilon}{\omega_{n}^{k}} \sum_{\nu=\mu}^{n} \omega_{\nu-1}^{k-1} N\left(a_{\nu}\right) .
$$

Now the first expression on the right, being a sum of $\mu-k$ terms independent of $n$, divided by $\omega_{n}^{k}$, approaches zero as $n \rightarrow \infty$. The second expression may be thrown into the form

$$
\frac{\epsilon}{\omega_{n}} \sum_{\nu=\mu}^{n}\left(\frac{\omega_{\nu-1}}{\omega_{n}}\right)^{k-1} N\left(a_{v}\right) \leqq \frac{\epsilon}{\omega_{n}} \sum_{\nu=\mu}^{n} N\left(a_{v}\right) \leqq \epsilon \alpha,
$$

the last inequality, in virtue of (4.2). Therefore

$$
0 \leqq \limsup _{n \rightarrow \infty} N[R(n, k)] \leqq \epsilon \alpha ;
$$

and this being valid for arbitrarily small $\epsilon$, shows that $R(n, k) \rightarrow 0$; and our lemma is proved.

5. The simple exponential theorems.

THEOREM I. If $\{\omega\}$ is any averaging sequence, and if $\{a\}$ is any sequence of elements of $\Re$ for which

$$
\lim _{n \rightarrow \infty} \frac{1}{\omega_{n}} \sum_{i=1}^{n} a_{i}=m, \quad \frac{1}{\omega_{n}} \sum_{i=1}^{n} N\left(a_{i}\right) \leqq \alpha<\infty,
$$


then

$$
\lim _{n \rightarrow \infty} \prod_{s=1}^{n}\left(1+\frac{a_{s}}{\omega_{n}}\right)=e^{m}
$$

Equations (4.6), (4.7), and Lemma III show that, for any fixed number $\mu$, the sum of the first $\mu$ terms in the expansion of the product in (5.1) approaches the sum of the first $\mu$ terms in the exponential series defining $e^{m}$ :

$$
\lim _{n \rightarrow \infty}\left[\sum_{k=0}^{\mu-1} p(n, k)-\sum_{k=0}^{\mu-1} \frac{m^{k}}{k !}\right]=0 .
$$

To establish (5.1) we shall use a method of majorants.

We have, from (4.7) and (4.5),

$$
\begin{aligned}
N[p(n, k)] & \leqq \frac{1}{\omega_{n}^{k}} \sum_{(i)}^{n} N\left(a_{i_{1}}\right) \cdots N\left(a_{i_{k}}\right) \\
& =\frac{1}{k !}\left[\frac{1}{\omega_{n}} \sum_{i=1}^{n} N\left(a_{i}\right)\right]^{k} \leqq \frac{\alpha^{k}}{k !},
\end{aligned}
$$

the last inequality on account of the last hypothesis.

Let $\epsilon>0$ be given, and chose a fixed $\mu$ so large that

$$
\sum_{k=\mu}^{\infty} \frac{N(m)^{k}}{k !}<\epsilon, \quad \sum_{k=\mu}^{\infty} \frac{\alpha^{k}}{k !}<\epsilon ;
$$

This is possible, because of the convergence of the series for $e^{N(m)}$ and $e^{\alpha}$. Then we have, once $n>\mu$,

$$
\prod_{s=1}^{n}\left(1+\frac{a_{s}}{\omega_{n}}\right)-e^{m}=\sum_{k=0}^{\mu-1}\left[p(n, k)-\frac{m^{k}}{k !}\right]+\sum_{k=\mu}^{n} p(n, k)-\sum_{k=\mu}^{\infty} \frac{m^{k}}{k !} ;
$$

and hence

$$
\begin{aligned}
N\left\{\prod_{s=i}^{n}\left(1+\frac{a_{s}}{\omega_{n}}\right)-e^{m}\right\} \leqq & \left\{\sum_{k=1}^{\mu-1} p(n, k)-\frac{m^{k}}{k !}\right\} \\
& +\sum_{k=\mu}^{\infty} \frac{\alpha^{k}}{k !}+\sum_{k=\mu}^{\infty} \frac{N(m)^{k}}{k !} \\
\leqq & N\left\{\sum_{k=1}^{\mu-1} p(n, k)-\frac{m^{k}}{k !}\right\}+2 \epsilon .
\end{aligned}
$$

Therefore

$$
0 \leqq \limsup _{n \rightarrow \infty} N\left\{\prod_{s=1}^{n}\left(1-\frac{a_{s}}{\omega_{n}}\right)-e^{m}\right\} \leqq 2 \epsilon ;
$$


and the validity of this for all $\epsilon>0$ suffices to prove (5.1).

Theorem II. The sequences $\{\omega\}$ and $\{a\}$ being as in Theorem I, let

$$
\left\{c_{n, i}\right\}=\left\{c_{n, 1}, c_{n, 2}, \cdots, c_{n, n}\right\}, \quad n=1,2, \cdots,
$$

be an infinite triangular array of elements of $\Re$ such that the quantity

$$
\phi_{n}=\frac{1}{\omega_{n}} \sum_{i=1}^{n} N\left(c_{n, i}\right) \rightarrow 0 \quad \text { as } n \rightarrow \infty .
$$

Then equation (5.1) subsists even if $a_{s}$ is replaced by $a_{s}+c_{n, 8}$. Moreover

$$
\prod_{s=1}^{n}\left(1+\frac{a_{s}+c_{n, s}}{\omega_{n}}\right)-\prod_{s=1}^{n}\left(1+\frac{a_{s}}{\omega_{n}}\right)=O\left(\phi_{n}\right) .
$$

Here the order symbol $O$ is based on the norm: if $\left\{f_{n}\right\}$ is a sequence of elements of $\Re, f_{n}=O\left(\phi_{n}\right)$ means that $N\left(f_{n}\right)=O\left(\phi_{n}\right)$.

By expansion,

$$
\begin{aligned}
\prod_{s=1}^{n}\left(1+\frac{a_{s}+c_{n, s}}{\omega_{n}}\right) & =\sum_{k=0}^{n} P(n, k) ; \\
P(n, 0) & =p(n, 0)=1, \\
P(n, k) & =\frac{1}{\omega_{n}^{k}} \sum_{(i)}^{n}\left(a_{i_{1}}+c_{n, i_{1}}\right) \cdots\left(a_{i_{k}}+c_{n, i_{k}}\right) \quad(1 \leqq k \leqq n) .
\end{aligned}
$$

Evidently, when $k \geqq 1$,

$$
P(n, k)=p(n, k)+q(n, k),
$$

where $q(n, k)$ is the sum of all those terms in the expansion of $P(n, k)$ which contain at least one factor $c_{n, i}$, the remaining factors being the $a$ 's. Grouping the terms in $q(n, k)$ according to the number, $r \geqq 1$, of factors $c_{n, i}$, we may write

$$
q(n, k)=\sum_{r=1}^{k} Q(n, k, r) ;
$$

and $Q(n, k, r)$ may be expressed as follows:

$$
Q(n, k, r)=\frac{1}{\omega_{n}^{k}} \sum_{(i)}^{k}\left[v_{i_{1}} \cdots v_{i_{k}}\right]
$$

where $\left[v_{i_{1}} \cdots v_{i_{k}}\right]$ is the sum of all the $C_{r}^{k}=k ! / r !(k-r) !$ different products obtained by replacing $r v$-symbols by $c$ 's and the remaining $k-r$ by $a$ 's, always with the corresponding subscripts and in the corresponding order.

It follows that 


$$
\begin{aligned}
N[Q(n, k, r)] & \leqq \frac{1}{\omega_{n}^{k}} \sum_{(i) \neq(j)}^{n} \sum_{(j)}^{n} N\left(c_{n, i_{1}}\right) \cdots N\left(c_{n, i_{r}}\right) N\left(a_{j_{1}}\right) \cdots N\left(a_{j_{k-r}}\right) \\
& \leqq \frac{1}{\omega_{n}^{r}} \sum_{(i)}^{n} N\left(c_{n, i_{1}}\right) \cdots N\left(c_{n, i_{r}}\right) \cdot \frac{1}{\omega_{n}^{k-r}} \sum_{(j)}^{n} N\left(a_{j_{1}}\right) \cdots N\left(a_{j_{k-r}}\right),
\end{aligned}
$$

where in the first summation $(i)=\left(i_{1}, \cdots, i_{r}\right)$ and $(j)=\left(j_{1}, \cdots, j_{k-r}\right)$ each run as in (4.3) except that no $i$ and $j$ are equal. In the last summation, this restriction is removed, thereby introducing certain non-negative terms. We have, further, by (4.5) and our hypothesis,

$$
\frac{1}{\omega_{n}^{r}} \sum_{(i)}^{n} N\left(c_{n, i_{1}}\right) \cdots N\left(c_{n, i_{r}}\right) \leqq \frac{\phi_{n}^{r}}{r !} .
$$

Similarly,

$$
\frac{1}{\omega_{n}^{r}} \sum_{(j)}^{n} N\left(a_{j_{1}}\right) \cdots N\left(a_{j_{k-r}}\right) \leqq \frac{\alpha^{k-r}}{(k-r) !} .
$$

It follows that

$$
\begin{aligned}
N[q(n, k)] & \leqq \sum_{r=1}^{k} N[Q(n, k, r)] \leqq \frac{1}{k !} \sum_{r=1}^{k} \frac{k !}{r !(k-r) !} \phi_{n}^{r} \alpha^{k-r} \\
& =\frac{1}{k !}\left[\left(\phi_{n}+\alpha\right)^{k}-\alpha^{k}\right],
\end{aligned}
$$

and therefore the norm of the left-hand member of (5.2) is less than or equal to

$$
\sum_{k=0}^{n}\left[\frac{\left(\phi_{n}+\alpha\right)^{k}}{k !}-\frac{\alpha^{k}}{k !}\right] \leqq \sum_{k=0}^{\infty}\left[\frac{\left(\phi_{n}+\alpha\right)^{k}}{k !}-\frac{\alpha^{k}}{k !}\right]=e^{\alpha}\left(e^{\phi_{n}}-1\right),
$$

which fact suffices to prove (5.2).

6. The composite exponential theorem. We now turn to the theorem which replaces (1.6) in the noncommutative case.

THEOREM III. Hypothesis: $\{\omega\}$ is an averaging sequence. $\{b\}$ is a sequence of elements of $\Re$ having the two properties

$$
\begin{array}{rlr}
N\left(b_{i}\right) \leqq \beta<\infty & (i=1,2, \cdots), \\
\lim _{\nu \rightarrow \infty} b_{i} b_{i+1} \cdots b_{i+\nu-1} & =B_{i} & \text { uniformly over } 0<i<\infty .
\end{array}
$$

$\{g\}$ is a sequence of elements of $\Re$ having the two properties

$$
\frac{1}{\omega_{n} ! i=1} \sum_{i=1}^{n} N\left(g_{i}\right) \leqq \gamma<\infty \quad(n=1,2, \cdots),
$$




$$
\lim _{i \rightarrow \infty} \frac{g_{i}}{\omega_{i}}=0 .
$$

Finally, $\{b\}$ and $\{g\}$ have the mutual property

$$
\lim _{n \rightarrow \infty} \frac{1}{\omega_{n}} \sum_{i=1}^{n} g_{i} B_{i+1}=M .
$$

Conclusion:

$$
\lim _{n \rightarrow \infty} \prod_{s=1}^{n}\left(b_{s}+\frac{g_{s}}{\omega_{n}}\right)=B_{1} e^{M} .
$$

Using a notation for "advancing factorial powers" of the calculus of finite differences, we shall write

$$
\begin{aligned}
& b_{i}^{(0)}=1 \\
& b_{i}^{(\lambda)}=b_{i} b_{i+1} \cdots b_{i+\lambda-1} \quad(i=1,2, \cdots) .
\end{aligned}
$$

And we shall set

$$
b_{i}^{(\lambda)}=B_{i}+f_{i}(\lambda) .
$$

Then (6.2) declares that, given any $\epsilon>0$, there exists an integer $\mu=\mu(\epsilon)$ independent of $i$, such that

$$
N\left[f_{i}(\lambda)\right]<\epsilon \quad \text { for all } \lambda \geqq \mu \text { and all } i=1,2, \cdots .
$$

Setting $\lambda=\mu$, we have, by (6.8), (6.9), (6.1), that

$$
\begin{aligned}
N\left(B_{i}\right) & \leqq N\left(b_{i}^{(\mu)}\right)+N\left(f_{i}(\mu)\right) \\
& \leqq N\left(b_{i}\right) N\left(b_{i+1}\right) \cdots N\left(b_{i+\lambda-1}\right)+\epsilon \\
& \leqq \beta^{\mu}+\epsilon,
\end{aligned}
$$

which shows that $\{B\}$ is a bounded sequence. This, in turn, shows that the aggregate $b_{i}^{(\lambda)}$ is bounded: If $\lambda<\mu$,

$$
N\left(b_{i}^{(\lambda)}\right) \leqq \beta^{\lambda}\left\{\begin{array}{lll}
\leqq \beta^{\mu} & \text { if } \quad \beta \geqq 1 \\
\leqq 1 & \text { if } \quad \beta<1 .
\end{array}\right.
$$

If $\lambda \geqq \mu$,

$$
N\left(b_{i}^{\lambda}\right) \leqq N\left(B_{i}\right)+N\left[f_{i}(\lambda)\right] \leqq \beta^{\mu}+2 \epsilon .
$$

We select an arbitrary common bound $\theta$, and write

$$
N\left(B_{i}\right) \leqq \theta, \quad N\left(b_{i}^{(\lambda)}\right) \leqq \theta \quad(i \geqq 1, \lambda \geqq 0) .
$$


It is now evident that the sequence $\{a\}$, where $a_{i}=g_{i} B_{i+1}$, verifies the hypothesis of Theorem I. We have (6.5); and also the fact that

$$
\frac{1}{\omega_{n}} \sum_{i=1}^{n} N\left(g_{i} B_{i+1}\right) \leqq \frac{1}{\omega_{n}} \sum_{i=1}^{n} \theta N\left(g_{i}\right) \leqq \theta \gamma .
$$

Consequently

$$
\lim _{n \rightarrow \infty} B_{1} \prod_{s=1}^{n}\left(1+\frac{g_{s} B_{s+1}}{\omega_{n}}\right)=B_{1} e^{M} .
$$

On the other hand, by simple expansion,

$$
\begin{aligned}
B_{1} \prod_{s=1}^{n}\left(1+\frac{g_{s} B_{s+1}}{\omega_{n}}\right) & =\sum_{k=0}^{n} p(n, k), \\
p(n, 0) & =B_{1}, \\
p(n, k) & =\frac{1}{\omega_{n}^{k}} \sum_{(i)}^{n} B_{1} g_{i_{1}} B_{i_{1}+1} \cdots g_{i_{k}} B_{i_{k}+1} \quad(k>0)
\end{aligned}
$$

and, similarly,

$$
\begin{aligned}
\prod_{s=1}^{n}\left(b_{s}+\frac{g_{s}}{\omega_{n}}\right) & =\sum_{k=0}^{n} u(n, k), \\
u(n, 0) & =b_{1}^{(n)}, \\
u(n, k) & =\frac{1}{\omega_{n}^{k}} \sum_{(i)}^{n} b_{1}^{\left(i_{1}-1\right)} g_{i_{1}} b_{i_{1}+1}^{\left(i_{2}-i_{1}-1\right)} g_{i_{2}} b_{i_{2}+1}^{\left(i_{s}-i_{2}-1\right)} \cdots g_{i_{k}} b_{i_{k}+1}^{\left(n-i_{k}\right)}(k>0) .
\end{aligned}
$$

If, therefore, we can prove that, for each $k$,

$$
\lim _{n \rightarrow \infty}[u(n, k)-p(n, k)]=0 ;
$$

and also exhibit a convergent series of positive constants, majorizing each of the series

$$
\begin{aligned}
& \sum_{k=0}^{n} N[p(n, k)], \\
& \sum_{k=0}^{n} N[u(n, k)]
\end{aligned}
$$

for every $n$, the proof of our theorem will be accomplished by the method of majorants used in Theorem I.

Such a majorant series is the power series for $\theta e^{\theta \gamma}$. That it majorizes (6.15) is seen on reference to the proof of Theorem I, with $a_{i}=g_{i} B_{i+1}$ and $\alpha=\theta \gamma$ (cf. (6.11)). That it majorizes (6.16) is shown by the following computation, 
using (4.5), (6.10), (6.4):

$$
N[u(n, k)] \leqq \frac{\theta^{k+1}}{\omega_{n}^{k}} \sum_{(i)}^{n} N\left(g_{i_{1}}\right) \cdots N\left(g_{i_{k}}\right) \leqq \frac{\theta^{k+1} \gamma^{k}}{k !} .
$$

The proof of (6.14) depends on Lemma IV, established in the following section.

7. The fourth lemma. In this section we assume the notation and hypothesis of Theorem III.

Lemma IV. For each value $k=0,1,2, \cdots$,

$$
\lim _{n \rightarrow \infty}[u(n, k)-p(n, k)]=0 .
$$

Let $v(n, k, r)$ be what becomes of $u(n, k)$ if in each term of its summation expression in (6.13), the first $r$ factorial powers of $b$ are replaced by $B$ 's with the same subscripts:

$$
\begin{aligned}
v(n, k, 0)= & u(n, k) ; \quad v(n, k, k+1)=p(n, k) ; \\
v(n, k, r)= & \frac{1}{\omega_{n}^{k}} \sum_{(i)}^{n} B_{1} g_{i_{1}} B_{i_{1}+1} \cdots g_{i_{r-1}} B_{i_{r-1}+1} \\
& \cdot g_{i_{r}} b_{i_{r}+1}^{\left(i_{r}+1-i_{r}-1\right)} \cdots g_{i_{k}} b_{i_{k}+1}^{\left(n-i_{k}\right)} \quad(1 \leqq r \leqq k) .
\end{aligned}
$$

Since obviously $u(n, k)-p(n, k)=\sum_{r=0}^{k}[v(n, k, r)-v(n, k, r+1)]$, (7.1) will be established once it is shown that, for $r=0,1, \cdots, k$,

$$
\lim _{n \rightarrow \infty}[v(n, k, r)-v(n, k, r+1)]=0 .
$$

In each term of the expression (7.2) for $v(n, k, r)$, replace the first $b_{i}^{(\lambda)}$ by $B_{i}+f_{i}(\lambda)$ of $(6.8)$ : If $r=0$, the first $b_{i}^{(\lambda)}$ is $b_{1}^{\left(i_{1}-1\right)}$; if $1 \leqq r \leqq k$, it is $b_{i_{r}+1}^{\left(i_{r+1}-i_{r}-1\right)}$. In all cases we obtain

$$
v(n, k, r)=v(n, k, r+1)+w(n, k, r)
$$

where $w(n, k, r)$ is obtained from $v(n, k, r)$ by the replacement process just described, only using $f_{i}(\lambda)$ instead of $B_{i}+f_{i}(\lambda)$. We have

$$
\begin{aligned}
w(n, k, 0) & =\frac{1}{\omega_{n}^{k}} \sum_{(i)}^{n} f_{1}\left(i_{1}-1\right) g_{i_{1}} b_{i_{1}+1}^{\left(i_{2}-i_{1}-1\right)} \cdots g_{i_{k}} b_{i_{k}+1}^{\left(n-i_{k}\right)} ; \\
w(n, k, k) & =\frac{1}{\omega_{n}^{k}} \sum_{(i)}^{n} B_{1} g_{i_{1}} B_{i_{1}+1} \cdots B_{i_{k-1}+1} g_{i_{k}} f_{i_{k}+1}\left(n-i_{k}\right) ; \\
w(n, k, r) & =\frac{1}{\omega_{n}^{k}} \sum_{(i)}^{n} B_{1} g_{i_{1}} B_{i_{1}+1} \cdots g_{i_{r-1}} B_{i_{r-1}+1} \\
g_{i_{r}} f_{i_{r}+1}\left(i_{r+1}-\right. & \left.i_{r}-1\right) g_{i_{r+1}} b_{i_{r+1}+1}^{\left(i_{r}+2-i_{r+1}-1\right)} \cdots g_{i_{k}} b_{i_{k}+1}^{\left(n-i_{k}\right)} \quad(0<r<k) .
\end{aligned}
$$


We shall call the factor $f_{i}(\lambda)$ in each term of $w(n, k, r+1)$ the pivotal factor of the term.

Let $\epsilon>0$ be given, and fix $\mu=\mu(\epsilon)$ as in (6.9). Then we may write

$$
w(n, k, r)=w^{\prime}(n, k, r)+w^{\prime \prime}(n, k, r),
$$

where $w^{\prime}(n, k, r)$ is the sum of all those terms in $w(n, k, r)$ whose privotal factors $f_{i}(\lambda)$ have $0 \leqq \lambda<\mu$, while $w^{\prime \prime}(n, k, r)$ is the sum of the terms whose pivotal factors $f_{i}(\lambda)$ have $\lambda \geqq \mu$.

From (7.5), (6.9), (6.10), (4.5), (6.3), we obtain

$$
N\left[w^{\prime \prime}(n, k, r)\right] \leqq \frac{\epsilon \theta^{k}}{\omega_{n}^{k}} \sum_{(i)}^{n} N\left(g_{i_{1}}\right) \cdots N\left(g_{i_{k}}\right) \leqq \frac{\epsilon \theta^{k} \gamma^{k}}{k !} .
$$

Consequently, if we can show that

$$
\lim _{n \rightarrow \infty} w^{\prime}(n, k, r)=0 \quad(r=0,1, \cdots, k),
$$

it will follow from (7.6) that $0 \leqq \lim \sup _{n \rightarrow \infty} N[w(n, k, r)] \leqq \theta^{k} \gamma^{k} \epsilon / k !$; and, $\epsilon$ being arbitrary, this will show that $w(n, k, r) \rightarrow 0$, and therefore, in view of (7.4), equation (7.3) will be established, and our lemma proved.

We shall use the following consequence of $(6.8),(6.10)$ :

$$
N\left[f_{i}(\lambda)\right] \leqq 2 \theta
$$

(6.9) is no longer available, since the present $\lambda<\mu$. Then we obtain from (7.5) and (6.10)

$$
\begin{aligned}
& N\left[w^{\prime}(n, k, 0)\right] \leqq \frac{\theta^{k}}{\omega_{n}^{k}} \sum_{(i)}^{n} N\left[f_{1}\left(i_{1}-1\right)\right] N\left(g_{i_{1}}\right) \cdots N\left(g_{i_{k}}\right) \\
& \leqq \frac{2 \theta^{k+1}}{\omega_{n}^{k}} \sum_{(i)}^{n}\left(i_{1} \leqq \mu\right) N\left(g_{i_{1}}\right) \cdots N\left(g_{i_{k}}\right), \\
& N\left[w^{\prime}(n, k, k)\right] \leqq \frac{\theta^{k}}{\omega_{n}^{k}} \sum_{(i)}^{n} N\left(g_{i_{1}}\right) \cdots N\left(g_{i_{k}}\right) N\left[f_{i_{k}+1}\left(n-i_{k}\right)\right] \\
& \leqq \frac{2 \theta^{k+1}}{\omega_{n}^{k}} \sum_{(i)}^{n}\left(i_{k}>n-\mu\right) N\left(g_{i_{1}}\right) \cdots N\left(g_{i_{k}}\right), \\
& N\left[w^{\prime}(n, k, r)\right] \leqq \frac{\theta^{k}}{\omega_{n}^{k}} \sum_{(i)}^{n} N\left(g_{i_{1}}\right) \cdots N\left(g_{i_{r}}\right) N\left[f_{i r+1}\left(i_{r+1}-i_{r}-1\right)\right] \\
& \cdot N\left(g_{i_{r}+1}\right) \cdots N\left(g_{k}\right) \\
& \leqq \frac{2 \theta^{k+1}}{\omega_{n}^{k}} \sum_{(i)}^{n}\left(i_{r+1}-i_{r} \leqq \mu\right) N\left(g_{i_{1}}\right) \cdots N\left(g_{i_{k}}\right) \\
& \quad(0<r<k) .
\end{aligned}
$$


The accents on the first summation signs in the first line on the right in each of these equations indicates the restriction that is made explicit in the second lines. Naturally, it results from the restriction $\lambda<\mu$ in the privotal factors.

It is easy to show that $w^{\prime}(n, k, 0)$ and $w^{\prime}(n, k, k)$ approach zero as $n \rightarrow \infty$ : The usual type of transformation (cf. (4.5)) applied to the last expression on the right in (7.8) gives

$$
\begin{aligned}
\frac{1}{\omega_{n}^{k}} \sum_{(i)}^{n}\left(i_{1} \leqq \mu\right) N\left(g_{i_{1}}\right) \cdots N\left(g_{i_{k}}\right) & =\frac{1}{\omega_{n}^{k}} \sum_{\nu=1}^{\mu} N\left(g_{\nu}\right) \sum_{(j)}^{n-v} N\left(g_{v+j_{1}}\right) \cdots N\left(g_{v+j_{k-1}}\right) \\
& \leqq \frac{1}{(k-1) !} \frac{1}{\omega_{n}} \sum_{\nu=1}^{\mu} N\left(g_{v}\right)\left[\frac{1}{\omega_{n}} \sum_{i=1}^{n} N\left(g_{i}\right)\right]^{k-1} \\
& \leqq \frac{\gamma^{k-1}}{(k-1) !} \frac{1}{\omega_{n}} \sum_{\nu=1}^{\mu} N\left(g_{v}\right) \rightarrow 0
\end{aligned}
$$

the last, on account of (3.2) and the fixed nature of $\mu$. Therefore $w^{\prime}(n, k, 0) \rightarrow 0$. Similarly,

$$
\begin{aligned}
\frac{1}{\omega_{n}^{k}} \sum_{(i)}^{n}\left(i_{k}>n-\mu\right) N\left(g_{i_{1}}\right) \cdots N\left(g_{i_{k}}\right) & =\frac{1}{\omega_{n}^{k}} \sum_{\nu=n-\mu+1}^{n} N\left(g_{v}\right) \sum_{(i)}^{\nu-1} N\left(g_{i_{1}}\right) \cdots N\left(g_{i_{k-1}}\right) \\
& \leqq \frac{\gamma^{k-1}}{(k-1) !} \frac{1}{\omega_{n}} \sum_{\nu=n-\mu+1}^{n} N\left(g_{\nu}\right) \rightarrow 0,
\end{aligned}
$$

but here the last statement requires (6.4) for its proof. We have

$$
\frac{1}{\omega_{n}} \sum_{\nu=n \rightarrow \mu+1}^{n} N\left(g_{\nu}\right)=\sum_{\nu=n-\mu+1}^{n} \frac{N\left(g_{\nu}\right)}{\omega_{\nu}} \cdot \frac{\omega_{\nu}}{\omega_{n}} \leqq \sum_{\nu=n-\mu+1}^{n} \frac{N\left(g_{\nu}\right)}{\omega_{\nu}}
$$

(cf. (3.1)). The last sum has the fixed number $\mu-1$ of terms; each approaches zero as $n \rightarrow \infty$, since then $\nu \rightarrow \infty$; at this point (6.4) gives the required result. Therefore $w^{\prime}(n, k, k) \rightarrow 0$.

It remains to study $w^{\prime}(n, k, r)$ when $(0<r<k)$. In the last expression on the right in (7.10), we may write

$$
\begin{aligned}
\frac{1}{\omega_{n}^{k}} \sum_{(i)}^{n}\left(i_{r+1}-i_{r} \leqq \mu\right) N\left(g_{i_{1}}\right) & \cdots N\left(g_{i_{k}}\right) \\
& =\sum_{\sigma=1}^{\mu} \frac{1}{\omega_{n}^{k}} \sum_{(i)}^{n}\left(i_{r+1}=i_{r}+\sigma\right) N\left(g_{i_{1}}\right) \cdots N\left(g_{i_{k}}\right) ;
\end{aligned}
$$

that is, we classify terms according to the difference $\sigma=i_{r+1}-i_{r}$, which may have all values from 1 to $\mu$, inclusive. Next, in each sum of terms corresponding to a fixed $\sigma$, we note that the factors fall into three groups, and write

$$
\frac{1}{\omega_{n}^{k}} \sum_{(i)}^{n}\left(i_{r+1}=i_{r}+\sigma\right) N\left(g_{i_{1}}\right) \cdots N\left(g_{i_{k}}\right)=\frac{1}{\omega_{n}^{2}} \sum_{\nu=r}^{n_{1}} G_{\nu} N\left(g_{\nu}\right) N\left(g_{\nu+\sigma}\right) H_{\nu, \sigma}
$$


where

$$
\begin{aligned}
G_{\nu} & =\frac{1}{\omega_{n}^{r-1}} \sum_{(i)}^{\nu-1} N\left(g_{i_{1}}\right) \cdots N\left(g_{i_{r-1}}\right), \\
H_{\nu, \sigma} & =\frac{1}{\omega_{n}^{k-r-1}} \sum_{(j)}^{n-\nu-\sigma} N\left(g_{\nu+\sigma+j_{1}}\right) \cdots N\left(g_{\nu+\sigma+j_{k-r-1}}\right),
\end{aligned}
$$

and $n_{1} \leqq n$ (actually, $n_{1}=n-k+r-\sigma+1$; but that is immaterial).

We have, by (4.5) and (6.3),

$$
\begin{aligned}
G_{\nu} & \leqq \frac{1}{(r-1) !}\left[\frac{1}{\omega_{n}} \sum_{i=1}^{\nu-1} N\left(g_{i}\right)\right]^{r-1} \leqq \frac{\gamma^{r-1}}{(r-1) !} ; \\
H_{\nu, \sigma} & \leqq \frac{1}{(k-r-1) !}\left[\frac{1}{\omega_{n}} \sum_{i=1}^{n-\nu-\sigma} N\left(g_{\nu+\sigma+i}\right)\right]^{k-r-1} \leqq \frac{\gamma^{k-r-1}}{(k-r-1) !} .
\end{aligned}
$$

Inserting these in (7.12), and allowing $\nu$ greater latitude,

$$
\begin{aligned}
\frac{1}{\omega_{n}^{k}} \sum_{(i)}^{n}\left(i_{r+1}\right. & \left.=i_{r}+\sigma\right) N\left(g_{i_{1}}\right) \cdots N\left(g_{i_{k}}\right) \\
& \leqq \frac{\gamma^{k-2}}{(r-1) !(k-r-1) !} \frac{1}{\omega_{n}^{2}} \sum_{v=1}^{n} N\left(g_{v}\right) N\left(g_{\nu+\sigma}\right) .
\end{aligned}
$$

If, therefore, we can prove that

$$
\lim _{n \rightarrow \infty} \frac{1}{\omega_{n}^{2}} \sum_{v=1}^{n} N\left(g_{v}\right) N\left(g_{v+\sigma}\right)=0,
$$

it will follow from (7.11) and (7.10) that $w^{\prime}(n, k, r) \rightarrow 0$, as desired.

Let $i(n)$ be the subscript of the (first) maximum $N\left(g_{i}\right)$ in the sequence

$$
N\left(g_{1}\right), N\left(g_{2}\right), \cdots, N\left(g_{n}\right) .
$$

We evidently have

$$
\frac{1}{\omega_{n}^{2}} \sum_{\nu=1}^{n} N\left(g_{v}\right) N\left(g_{v+\sigma}\right) \leqq \frac{N\left(g_{i(n)}\right)}{\omega_{n}} \frac{1}{\omega_{n}} \sum_{\nu=1}^{n} N\left(g_{v+\sigma}\right) \leqq \frac{N\left(g_{i(n)}\right)}{\omega_{n}} \gamma .
$$

Now $i(n)$ increases, if it changes at all, as $n$ increases. First possibility: $i(n)$ is bounded as $n \rightarrow \infty$; then $N\left[g_{i(n)}\right] / \omega_{n} \rightarrow 0$. Second possibility: $i(n) \rightarrow \infty$; then

$$
\frac{N\left[g_{i(n)}\right]}{\omega_{n}}=\frac{N\left[g_{i(n)}\right]}{\omega_{i(n)}} \cdot \frac{\omega_{i(n)}}{\omega_{n}} \leqq \frac{N\left[g_{i(n)}\right]}{\omega_{i(n)}} \rightarrow 0,
$$

on account of (6.4). Thus (7.13) is proved in all cases; and therewith our lemma.

8. An extension of the composite exponential theorem. In this section we 
shall derive a theorem which bears the same relation to Theorem III as Theorem II does to Theorem I.

THEOREM IV. If $\{\omega\},\{b\},\{g\}$ fulfill the hypothesis of Theorem III, and if the aggregate $\left\{c_{n, s}\right\}$ verifies the hypothesis of Theorem II, then equation (6.6) subsists even when, in this equation, $g_{\mathrm{s}}$ is replaced by $g_{8}+c_{n, s}$. Moreover

$$
\prod_{s=1}^{n}\left(b_{s}+\frac{g_{s}+c_{n, s}}{\omega_{n}}\right)-\prod_{s=1}^{n}\left(b_{s}+\frac{g_{s}}{\omega_{n}}\right)=O\left(\phi_{n}\right) .
$$

The proof is altogether similar to that of Theorem II. Write

$$
\prod_{s=1}^{n}\left(b_{s}+\frac{g_{s}+c_{n, s}}{\omega_{n}}\right)=\sum_{k=0}^{n} U(n, k),
$$

where $U(n, k)$ is what $u(n, k)$ becomes if in (6.13) each factor $g_{i}$ is replaced by the corresponding $g_{i}+c_{n, i}$. We have

$$
U(n, k)=u(n, k)+V(n, k),
$$

where $V(n, k)$ is the sum of all those terms in the expansion of $U(n, k)$ which contain at least one factor $c_{n, i}$, the remaining factors being the $g$ 's and $b$ 's. Grouping terms according to the number $r$ of factors $c_{n, i}$, we have

$$
\begin{aligned}
V(n, k) & =\sum_{r=1}^{k} V(n, k, r), \\
V(n, k, r) & =\frac{1}{\omega_{n}^{k}} \sum_{(i)}^{n}\left[b_{1}^{\left(i_{1}-1\right)} v_{i_{1}} b_{i_{1}+1}^{\left(i_{2}-i_{1}-1\right)} v_{i_{2}} \cdots v_{i_{k}} b_{i_{k}+1}^{\left(n-i_{k}\right)}\right],
\end{aligned}
$$

where the bracket $\left[\cdots v_{i_{1}} \cdots v_{i_{k}} \cdots\right]$ is the sum of all $C_{r}^{k}$ products obtained by replacing $r v$-symbols by $c$ 's and the remaining $k-r v$ 's by $g$ 's, always with the corresponding subscripts and with maintenance of order.

We derive as usual

$$
\begin{aligned}
N[v(n, k, r)] & \leqq \frac{\theta^{k+1}}{\omega_{n}^{k}} \sum_{(i) \neq(i)}^{n} \sum_{n}^{n} N\left(c_{n, i_{1}}\right) \cdots N\left(c_{n, i_{r}}\right) N\left(g_{j_{1}}\right) \cdots N\left(g_{j_{k-r}}\right) \\
& \leqq \frac{\theta^{k+1}}{r !(k-r) !}\left[\sum_{i=1}^{n} N\left(c_{n, i}\right)\right]^{r}\left[\sum_{j=1}^{n} N\left(g_{j}\right)\right]^{k-r} \\
& \leqq \frac{\theta^{k+1}}{k !} \frac{k !}{r !(k-r) !} \phi_{n}^{r} \gamma^{k-r}, \\
N[V(n, k)] & \leqq \frac{\theta^{k+1}}{k !} \sum_{r=1}^{k} C_{r}^{k} \phi_{n}^{r} \gamma^{k-r} \\
& =\frac{\theta^{k+1}}{k !}\left[\left(\phi_{n}+\gamma\right)^{k}-\gamma^{k}\right] .
\end{aligned}
$$


Therefore the norm of the left-hand side of (8.1) is not greater than

$$
\sum_{k=0}^{\infty} \frac{\theta^{k+1}}{k !}\left[\left(\phi_{n}+\gamma\right)^{k}-\gamma^{k}\right]=\theta e^{\theta \nu}\left(e^{\theta \phi_{n}}-1\right) ;
$$

which furnishes the conclusion of the theorem.

9. A generalization of the exponential theorems. We conclude with a straightforward generalization of the composite exponential theorem and its extension. While it is established by an almost trivial step-by-step modification of the proofs of Theorems III and IV, it is of sufficient usefulness in probability to warrant an explicit formulation.

The generalization consists in replacing the sequence $\left\{b_{i}\right\}$ of the preceding theorems by the infinite triangular array $\left\{b_{n, i}\right\}: n=1,2, \cdots ; i=1, \cdots$, $n$. This aggregate of elements of our Banach algebra satisfies the two following conditions, corresponding to (6.1) and (6.2):

$$
\begin{aligned}
N\left(b_{n, i}\right) & \leqq \beta<\infty \quad(n=1,2, \cdots ; 1 \leqq i \leqq n) . \\
b_{n, i}^{(v)} & =b_{n, i} b_{n, i+1} \cdots b_{n, i+\nu-1} \rightarrow B_{i}
\end{aligned}
$$

uniformly over $i$ as $\nu$ and $n$ become infinite independently, but with $i+\nu-1 \leqq n$. In other words, writing

$$
b_{n, i}^{(\lambda)}=B_{i}+f_{i}(n, \lambda),
$$

we are assuming that, given any positive number $\epsilon$, there exists an integer $\mu=\mu(\epsilon)$, independent of $i$, such that

$$
N\left[f_{i}(n, \lambda)\right]<\epsilon \quad \text { for all } \lambda \geqq \mu \text { and } n \geqq \nu+i-1 .
$$

THEOREM V. If $\left\{b_{n, i}\right\}$ satisfies the conditions (9.1) and (9.2), and if the sequences $\{\omega\},\{g\},\{B\}$ satisfy the hypotheses (6.3), (6.4), (6.5), of Theorem III, then the conclusion corresponding to (6.6) follows, namely

$$
\lim _{n \rightarrow \infty} \prod_{s=1}^{n}\left(b_{n, s}+\frac{g_{s}}{\omega_{n}}\right)=B_{1} e^{M} .
$$

First, the boundedness is established:

$$
N\left(B_{i}\right) \leqq \theta ; \quad N\left(b_{n, i}^{(\lambda)}\right) \leqq \theta \quad(\theta<\infty) ;
$$

the first applying for all $i=1,2, \cdots$; the second, for all $n=1,2, \cdots$, and all $i, \lambda$ for which $i+\lambda-1 \leqq n$. This is done as in the proof of (6.10), with (9.1) and (9.3) replacing (6.1) and (6.8).

From there on, one simply replaces every $b_{i}$ or $b_{i}^{(\lambda)}$ by $b_{n, i}$ or $b_{n, i}^{(\lambda)}$. Equation (6.12) is unchanged, while (6.13) is modified as stated; every factor such as $b_{n, 2_{2}+1}^{\left(i_{3}-1 i_{2}-1\right)}$ is meaningful, since clearly $\left(i_{2}+1\right)+\left(i_{3}-i_{2}-1\right)=i_{3} \leqq n$. The corresponding observation applies in (7.2) and (7.5), the pivotal factor $f_{i_{r}+1}\left(i_{r+1}-i_{r}-1\right)$ being replaced by $f_{i_{r}+1}\left(n, i_{r+1}-i_{r}-1\right)$ in the latter. With 
regard to (7.6), making $\lambda \geqq \mu$ (that is, $i_{r+1}-i_{r}-1 \geqq \mu$ ) automatically insures that $n$ is large enough to bring (9.4) into force. Finally, once equations (7.8), (7.9), (7.10) have been observed to hold, the remaining rather complicated steps proceed exactly as before since they no longer involve the modified $b$ quantities.

By a precisely similar modification of some of the expressions in the proof of Theorem IV, we generalize it, and obtain the following last and most general form of the exponential limiting product theorem of the present paper:

Theorem VI. If $\{\omega\},\left\{b_{n, i}\right\},\{g\}$ fulfill the hypothesis of Theorem V and $\left\{c_{n, 8}\right\}$ that of Theorem II, then

$$
\lim _{n \rightarrow \infty} \prod_{s=1}^{n}\left(b_{n, s}+\frac{g_{s}+c_{n, s}}{\omega_{n}}\right)=B_{1} e^{M} .
$$

Moreover, equation (8.1) applies with $b_{8}$ replaced by $b_{n, 8 .}$.

Columbia University,

NEw York, N. Y. 